\title{
Industrialisierung von Softwareunternehmen durch Arbeitsteilung: Einzelfall oder Trend?
}

\author{
Christian M. Wolf, Alexander Benlian, Thomas Hess \\ Institut für Wirtschaftsinformatik und Neue Medien, \\ Ludwig-Maximilians-Universität München
}

\section{Einleitung}

In letzter Zeit ließ sich in verschiedenen Branchen (z. B. Automobil, Chemie) eine zunehmende Auflösung der Wertschöpfungsketten beobachten. Diese manifestiert sich in einer verringerten Bedeutung hierarchischer Koordination zugunsten von marktlichen oder hybriden Koordinationsformen und somit einer Industrialisierung im Sinne einer zunehmenden zwischenbetrieblichen Arbeitsteilung (Picot und Baumann 2009, S. 75). Da Informations- und Kommunikationstechnologien Treiber bzw. Enabler dieses organisationstheoretischen Phänomens sind (Picot et al. 2008) und diese gleichzeitig einen wichtigen Betrachtungsgegenstand der an der Nahtstelle von Technologie und Wirtschaft angesiedelten Wirtschaftsinformatik (Mertens und Heinrich 2002; Mertens et al. 2005; Wigand et al. 2003) darstellen, schlagen Picot und Baumann (2009, S. 78) der Wirtschaftsinformatikforschung vor, organisationsbezogenen Fragestellungen, wie „den Einflussgrößen und Einsichten zu organisatorischen Phänomenen und Beziehungen" (Picot und Baumann 2008, S. 72) eine größere Aufmerksamkeit zu widmen. Diese Anregung wollen wir mit einer Betrachtung der Entwicklung der zwischenbetrieblichen Arbeitsteilung von Softwareunternehmen, die bzw. deren Produkte einen weiteren wichtigen Betrachtungsgegenstand der Wirtschaftsinformatik darstellen, im Rahmen des vorliegenden Beitrags aufgreifen.

Um die damit verbundene konkrete Forschungsfrage zu beantworten, ob und in welchen Bereichen der Wertschöpfungskette sich die zunehmende zwischenbetriebliche Arbeitsteilung bei Softwareunternehmen manifestiert, führten wir eine Multifallstudienuntersuchung bei mittelständischen Herstellern erklärungs- und anpassungsbedürftiger betriebswirtschaftlicher Standardsoftware aus Deutschland durch. Unser erklärtes Ziel war es dabei, die Entwicklung der Arbeitsteilung auf den einzelnen Wertschöpfungsstufen detailliert zu beschreiben, um so die Basis für eine Erklärung der festgestellten Entwicklungen zu liefern.

Die Durchführung und die Ergebnisse unserer Studie sind nachfolgend dargestellt. Dazu erfolgt in Kapitel 2 zunächst eine kurze Einordnung der zwischenbet- 
rieblichen Arbeitsteilung als Element der Industrialisierung. Im 3. Kapitel stellen wir die Konzeption unserer Untersuchung und dabei insbesondere den zur Anwendung gekommenen Untersuchungsrahmen vor, der in Kapitel 4 für drei erhobene Fälle ausgefüllt wird. Wir schließen den Beitrag in Kapitel 5 mit einem Fazit.

\section{Zwischenbetriebliche Arbeitsteilung als Element der Industrialisierung}

In der Literatur wird zwischen horizontaler und vertikaler Arbeitsteilung unterschieden. Wenn Aufgaben auf mehrere organisatorische Einheiten verteilt werden, wobei jede Einheit dieselbe Teilaufgabe erfüllt, liegt horizontale Arbeitsteilung vor. Wenn eine Gesamtaufgabe hingegen in Teilaufgaben unterschiedlicher Art zerlegt wird, die dann von verschiedenen spezialisierten Akteuren wahrgenommen werden, spricht man von vertikaler Arbeitsteilung. Demnach führt nur die vertikale Arbeitsteilung zu inhaltlichen Merkmalsunterschieden der zu erfüllenden Teilaufgaben und somit zu einer Spezialisierung der beteiligten Akteure (Zäpfel 1989, S. 157).

Außerdem kann in innerbetriebliche und zwischenbetriebliche Arbeitsteilung unterschieden werden. Dabei bedeutet erstere, dass die Teilaufgaben in spezialisierten Organisationseinheiten innerhalb eines Unternehmens (z. B. Stellen, Abteilungen) gebündelt werden. Zweite hingegen bedeutet, dass die Teilaufgaben in spezialisierten Unternehmen gebündelt werden.

In unserer Untersuchung fokussierten wir uns auf die zwischenbetriebliche vertikale Arbeitsteilung. Diese Frage wird heute häufig dem Themenkomplex der Industrialisierung zugeordnet. Neben einer verstärkten Standardisierung von Prozessen und Produkten sowie einer Zunahmen an Automatisierung (Budäus 1990, S. 5; Heinen 1991, S. 10; Schweitzer 1991, S. 19-21) lässt sich solch ein steigender Grad an (zwischenbetrieblicher) Arbeitsteilung als dritte der zueinander in Beziehung stehenden Elemente des Managementkonzepts der Industrialisierung einordnen (Buxmann et al. 2008, S. 131).

In letzter Zeit finden sich vermehrt Hinweise, dass auch Softwareunternehmen einer Industrialisierung unterzogen sind (Buxmann et al. 2008, S. 130; Fischer 2007; Hess et al. 2007; Hildenbrand et al. 2007; Kilian-Kehr et al. 2007; Meyer und Stobbe 2007; Wolf et al. 2008). In der aktuellen Diskussion werden Informationsund Kommunikationstechnologien (IKT) als die technologischen Treiber der Industrialisierung angesehen, sodass man in diesem Zusammenhang auch von einer Industrialisierung durch IKT spricht. Diese kann sämtliche Branchen betreffen, in deren Unternehmen es durch den Einsatz von IKT zu höheren Graden an Standardisierung, Arbeitsteilung und/oder Automatisierung kommt. Davon abzugrenzen ist die Industrialisierung der IKT selbst, die vorliegt, wenn die Hersteller von IKT durch eine oder mehrere der genannten Ausprägungsformen industrialisiert werden. Softwareunternehmen sind interessanterweise von beiden Varianten be- 
troffen. Zum einen zählen ihre Produkte zur IKT und können somit zur Industrialisierung der Anwenderunternehmen durch IKT beitragen. Auf Grund Ihrer Zugehörigkeit zur IKT-Branche können Softwareunternehmen zum anderen selbst Gegenstand einer Industrialisierung der IKT sein. Letztgenannten Fall haben wir anhand der Ausprägungsform der Arbeitsteilung in unserer vorliegenden Untersuchung vertieft betrachtet.

\section{Konzeption der Fallstudienuntersuchung}

Unsere Untersuchung orientiert sich hinsichtlich Design, Ablauf und Auswertung an dem Konzept und den Empfehlungen für Fallstudienuntersuchungen von Yin (2003). Um die Vergleichbarkeit mit vorausgehenden Studien (z. B. Wolf et al. (2008)) zu gewährleisten, fokussierten wir uns auf Anbieter von erklärungs- und anpassungsbedürftiger betriebswirtschaftlicher Standardsoftware. Des Weiteren erfolgte eine Einschränkung hinsichtlich der Software-Architektur auf monolithische Systeme, da die monolithische Architektur in der zu betrachtenden Vergangenheit dominant war und neuere Konzepte, wie hochgradig modulare, auf WebServices beruhende serviceorientierte Architekturen die zwischenbetriebliche Arbeitsteilung stark hätten beeinflussen und somit die Vergleichbarkeit der Fälle hätten verfälschen können.

Leitfadengestützte Experteninterviews bildeten den Ausgangs- und Mittelpunkt der Erhebung. Die Interviews waren teilstandardisiert, d. h. es wurde auf einen Fragenkatalog und Gesprächsleitfaden zurückgegriffen, um eine Struktur in die Gespräche zu bringen. Obwohl alle notwendigen Daten durch Interviews erhoben werden konnten, wurde zur Sicherung der Validität der Untersuchung, wie von Yin (2003, S. 98-99) empfohlen, im Sinne einer Datentriangulation auf eine ergänzende Dokumentenanalyse zurückgegriffen und die erarbeiteten Fallbeschreibungen wurden durch die Informanden durchgesehen. Als Datenquellen der Dokumentenanalyse dienten insbesondere die Internetauftritte sowie zur Verfügung gestellte interne Dokumente der Fallstudienunternehmen.

Zur adäquaten Untersuchung der Forschungsfrage ist ein geeigneter Rahmen, anhand dessen die zwischenbetriebliche Arbeitsteilung adäquat untersucht und visualisiert werden kann, notwendig. Bezugspunkte dieses Untersuchungsrahmens sind die in Abbildung 1 dargestellten, generischen Wertschöpfungsstufen von Herstellern erklärungs- und anpassungsbedürftiger betriebswirtschaftlicher Standardsoftware.

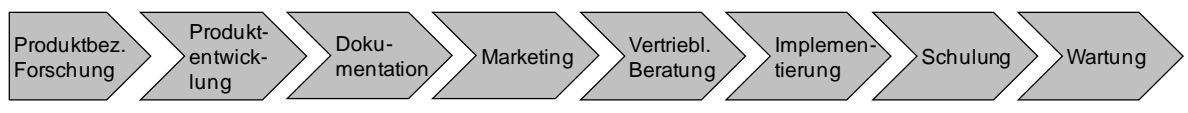

Abbildung 1: Generische Wertschöpfungsstufen von Softwareherstellern 
Ziel ist es, für jede Wertschöpfungsstufe den Grad an zwischenbetrieblicher Arbeitsteilung aus der Sicht des jeweiligen Fallstudienunternehmens herauszuarbeiten. Den idealtypischen Koordinationsformen von Williamson (1991, S. 269) folgend, beantworten wir damit die Frage, ob die Teilaktivitäten einer Wertschöpfungsstufe zu einem bestimmten Betrachtungszeitpunkt vom Fallstudienunternehmen selbst übernommen (Hierarchie), von Partnerunternehmen in hybrider Koordination erbracht, fremd über den Markt koordiniert oder in verschiedenen Koordinationsformen durchgeführt wurden.

Dazu greifen wir auf eine Visualisierung in Anlehnung an die Organisationsprozessdarstellung (Binner 1987) zurück, die unter dem Namen „Swimlane“ in vielen Prozessmodellierungs-Tools angeboten wird. Jede Wertschöpfungsstufe wird dabei entsprechend der in den Fallstudien ermittelten zwischenbetrieblichen Arbeitsteilung bei ihrer Durchführung auf oder zwischen einer der „Swimlanes“ Hierarchie, Hybrid oder Markt platziert. Befindet sich eine Wertschöpfungsstufe ausschließlich auf der „Swimlane“ Hierarchie, werden sämtliche dazugehörige Teilaktivitäten rein vom jeweiligen Fallstudienunternehmen selbst durchgeführt und es liegt keine zwischenbetriebliche Arbeitsteilung vor. Eine Platzierung auf der Begrenzung zwischen Hierarchie und Hybrid bedeutet hingegen einen höheren Grad an zwischenbetrieblicher Arbeitsteilung, da eine oder mehrere Teilaktivitäten in der Koordinationsform Hybrid und somit arbeitsteilig erbracht werden.

Der höchste Grad an zwischenbetrieblicher Arbeitsteilung aus der Sicht des jeweiligen Fallstudienunternehmens liegt - bezogen auf eine Wertschöpfungsstufe - vor, wenn sich diese vollständig auf einer der „Swimlanes“ Hybrid oder Markt oder auf der Grenze dazwischen befindet, da dann keine der jeweiligen Teilaktivitäten vom Fallstudienunternehmen selbst übernommen wird. Vergleicht man den ausgefüllten Untersuchungsrahmen zweier verschiedener Zeitpunkte, so lässt sich feststellen, ob und auf welchen Wertschöpfungsstufen es beim jeweiligen Fallstudienunternehmen zu einer Industrialisierung im Sinne einer zunehmenden zwischenbetrieblichen Arbeitsteilung gekommen ist.

\section{$4 \quad$ Ergebnisse der Fallstudienuntersuchung}

\subsection{Fallstudie 1}

Die Fallstudie wurde im Mai 2009 bei einem eigenständigen, mittelständischen Standardsoftwarehersteller mittels einer Dokumentenanalyse sowie eines gut einstündigen Telefoninterviews mit der Leiterin des Bereichs Marketing/PR erhoben. Das Produkt, dessen Wertschöpfungskette betrachtet wurde, ist eine ERP-Lösung für kleine und mittelgroße Unternehmen, welche die Bereiche Warenwirtschaft und Produktion, Finanzbuchhaltung, Geschäftsanalyse, Personalmanagement und Kundenmanagement abdeckt und dabei helfen soll, die kaufmännischen und betriebswirtschaftlichen Anforderungen von Unternehmen aus den Bereichen Han- 
del, Industrie sowie Dienstleistung zu lösen. Neben der aktuellen Situation wurde vom Unternehmen als zu betrachtender Zeitpunkt in der Vergangenheit das Jahr 2005 festgelegt.

Die produktberogene Forschung wurde 2009 grundsätzlich von internen Mitarbeitern durchgeführt, wobei für bestimmte, den Funktionsumfang erweiternde Schnittstellenprodukte Kooperationspartner miteinbezogen wurden. 2005 hingegen wurde die produktbezogene Forschung ausschließlich intern durchgeführt. Somit lag 2005 im Gegensatz zu 2009 sowohl hierarchische als auch hybride Koordination vor.

Die Produktentwicklung des Kernprodukts wurde zu beiden Betrachtungszeitpunkten innerhalb des Unternehmens und somit in der idealtypischen Koordinationsform Hierarchie durchgeführt. Allerdings gab es 2009 die bei der produktbezogenen Forschung bereits erwähnten Schnittstellenprodukte, die den Leistungsumfang des Kernprodukts erweiterten und auch von Kooperationspartnern in der Koordinationsform Hybrid entwickelt wurden.

Die Wertschöpfungsstufe Dokumentation wurde 2009 wie 2005 im Unternehmen intern, also in der Koordinationsform Hierarchie, von spezialisierten Mitarbeitern aus dem Unternehmensbereich Qualitätsmanagement durchgeführt.

Die generischen Marketingaktivitäten wie Messen, Anzeigen, Online-Medien, Branding oder Brand-Kampagnen wurden sowohl 2009 als auch 2005 intern und somit in der Koordinationsform Hierarchie durchgeführt.

Das Unternehmen verfolgte 2009 wie 2005 grundsätzlich ein indirektes Vertriebsmodell: Endkunden konnten das Produkt nicht direkt vom Fallstudienunternehmen, sondern ausschließlich über teilweise auf bestimmte Produktlinien oder Branchen spezialisierte Vertriebspartner und Systemhäuser beziehen, mit denen eine langfristige Kooperationsbeziehung (teilweise bis zu 16 Jahren) bestand, sodass auf der Wertschöpfungsstufe vertriebliche Beratung zu beiden Betrachtungszeitpunkten die Koordinationsform Hybrid vorlag. Des Weiteren gab es 2009 im Gegensatz zu 2005 auch eigene Mitarbeiter des Fallstudienunternehmens im Bereich vertriebliche Beratung, welche die Partner auf Anfrage bei Kundenpräsentationen unterstützen, sodass 2009 neben hybrider auch hierarchische Koordination vorlag.

Die Wertschöpfungsstufe der Implementierung wurde zu beiden Betrachtungszeitpunkten in der Koordinationsform Hybrid vollständig von den schon im Bereich vertriebliche Beratung genannten Kooperationspartnern durchgeführt, ohne dass diese - wie es 2009 auf der vorangegangenen Wertschöpfungsstufe der Fall war - von internen Mitarbeitern des Fallstudienunternehmens unterstützt wurden.

Grundsätzlich wurde die Schulung insbesondere bei Neukunden zu beiden Betrachtungszeitpunkten von den bereits im Bereich der vertrieblichen Beratung und der Implementierung tätigen Kooperationspartnern und somit in der Koordinationsform Hybrid durchgeführt. Diese schulten so genannte Key-User des Kunden im Umgang mit der Software, die dann wiederum intern weiterschulten. Lediglich bei bestimmten neuen Releases oder Änderungen des Softwareprodukts, z. B. im Bereich Finanzwesen oder Lohnbuchhaltung, die ein Partner ggfs. aus inhaltlicher 
Sicht nicht leisten konnte, bot das Fallstudienunternehmen 2009 auch eigene Schulungen an, die es 2005 noch überhaupt nicht gab.

Im Bereich des betrachteten Softwareprodukts wurde die Wartung sowohl 2009 als auch schon 2005 vollständig von den Kooperationspartnern des Fallstudienunternehmens übernommen und somit in der Koordinationsform Hybrid durchgeführt.

Zusammenfassend ergeben sich für 2005 und 2009 die in Abbildung 2 dargestellten Ausprägungen der zwischenbetrieblichen Arbeitsteilung.

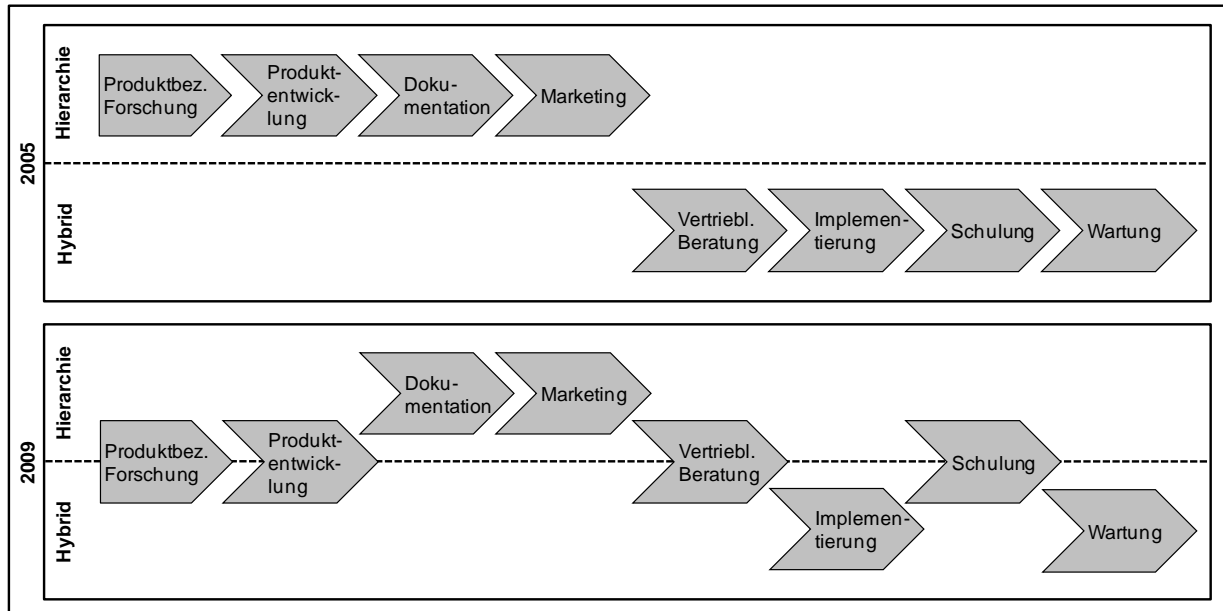

Abbildung 2: Zwischenbetriebliche Arbeitsteilung der Fallstudie 1 im Vergleich

\subsection{Fallstudie 2}

Die Fallstudie wurde im Mai 2009 bei einem der führenden mittelständischen Beratungs- und Softwareunternehmen für integrierte ERP-Lösungen im deutschsprachigen Raum mittels einer Dokumentenanalyse sowie eines zweistündigen persönlichen Interviews mit dem Leiter des Bereichs Business Development erhoben. Das Produkt, dessen Wertschöpfungsstruktur betrachtet wurde, ist ein modular aufgebautes, mehrsprachiges, mandanten- und mehrserverfähiges ERP-System für Kunden aus dem Bereich der Einzel-, Auftrags- und Variantenfertigung. Neben der aktuellen Situation wurde als zu betrachtender Zeitpunkt in der Vergangenheit das Jahr 2000 festgelegt.

Die Wertschöpfungsstufen produktberogene Forschung und Produktentwicklung wurden vom Fallstudienunternehmen nicht getrennt, sondern als eine integrierte Stufe namens produktberogene Forschung und Produktentwicklung betrachtet. Neue Produktideen und aktuelle Kundenbedürfnisse im Sinne der produktbezogenen Forschung wurden zu beiden Betrachtungszeitpunkten von eigenen Mitarbeitern ermittelt, festgesetzt und an die Entwicklungsabteilung gegeben. Innerhalb der Pro- 
duktentwicklung grenzte das Fallstudienunternehmen ab zwischen der eigentlichen Applikations-Entwicklung im Rahmen derer die vom Markt gewünschten Funktionalitäten im Softwareprodukt umgesetzt wurden und der technischen Entwicklung, die sich damit befasste, mit welchen Programmier-Tools die eben genannte Applikations-Entwicklung des Softwareprodukts zukunftsweisend umgesetzt werden konnte. Die technische Entwicklung und die Applikations-Entwicklung des Kernprodukts wurden 2000 vollständig von unternehmensinternen Mitarbeitern durchgeführt, sodass die Koordinationsform Hierarchie vorlag. 2009 existierten im Gegensatz zu 2000 außerdem verschiedene Möglichkeiten, externe Programme wie z. B. Outlook, Excel oder Word aus dem Softwareprodukt heraus anzusteuern oder bestimmte Basisfunktionalitäten der für das Softwareprodukt verwendeten Microsoft SQL-Datenbank, wie z. B. grafische Darstellungen, zu nutzen, die aus Nutzersicht wie ein integraler Bestandteil aussahen. Da dies jedoch Standardfunktionen der für die Entwicklung verwendeten Microsoft-Technologien waren, können sie nicht als von externen Transaktionspartnern hinzugekaufte Programmodule im Sinne dieser Untersuchung verstanden werden, die auf die Koordinationsform Hybrid hindeuten würden. Jedoch die 2009 verfügbaren und in das System integrierbaren Module für Personalmanagement und Frachtpapiere/Zollabwicklung wurden von externen Softwarepartnern erstellt, sodass neben der hierarchischen Koordination auf der Wertschöpfungsstufe produktberogene Forscbung und Produktentwicklung 2009 auch hybride Koordination vorlag.

Um bildlich gesprochen in einem Kopf sowohl das Fach-, Architektur-, Codier- und Dokumentationswissen zu haben, wurde die Dokumentation zu beiden Betrachtungszeitpunkten von den im Bereich produktbezogene Forschung und Produktentwicklung tätigen internen Mitarbeitern und somit in der Koordinationsform Hierarchie durchgeführt.

2009 verfügte das Fallstudienunternehmen über eine eigene Marketingabteilung, welche die Pressearbeit und CRM-Aktivitäten wie Kundenkontaktpflege, Interessentendatenbankpflege oder Telefonmarketing durchführte sowie das von einer externen Agentur erstellte Markenbild (Logos, Layouts, Briefbögen, Vorlagen für Stellenanzeigen usw.) über Werbung am Markt kommunizierte. 2000 gab es diese Zusammenarbeit mit der externen Agentur noch nicht, sondern auch die später von der Agentur übernommenen Aktivitäten wurden noch intern durchgeführt. Somit lag auf der Wertschöpfungsstufe Marketing 2009 sowohl hierarchische als auch hybride Koordination und 2000 hingegen rein hierarchische Koordination vor.

2009 fuhr das Fallstudienunternehmen für den deutschen Markt ein rein direktes Vertriebsmodell und für alle anderen Länder ein indirektes Vertriebsmodell mit jeweils wenigen, ausgewählten und qualitativ hochwertigen Franchisenehmern, an denen auch Minderheitsbeteiligungen bestanden. 2000 hingegen wurde - auf allen DACH-Märkten - noch viel stärker auf Partner gesetzt, was sich allerdings nicht bewährt hatte, da das Produkt oftmals nicht ordnungsgemäß verkauft und betreut wurde. Hinsichtlich der idealtypischen Koordinationsformen lag auf der Wert- 
schöpfungsstufe vertriebliche Beratung somit zu beiden Betrachtungszeitpunkten sowohl hierarchische als auch hybride Koordination vor.

Die Wertschöpfungsstufe der Implementierung wurde, wie schon die vertriebliche Beratung, 2009 auf dem deutschen Markt vom Fallstudienunternehmen selbst und somit in der Koordinationsform Hierarchie und auf ausländischen Märkten von den bereits erwähnten wenigen, ausgewählten und qualitativ hochwertigen Franchisenehmern erbracht. Auch 2000 wurde die Implementierung auf ausländischen Märkten von Partnern und somit in der Koordinationsform Hybrid erbracht, allerdings führten Partnerunternehmen auch in Deutschland Implementierungsprojekte durch. Hinsichtlich der idealtypischen Koordinationsformen lag somit zu beiden Betrachtungszeitpunkten wiederum sowohl hierarchische als auch hybride Koordination vor.

Da die mit dem Softwareprodukt gelösten Probleme in der Regel sehr kundentypisch seien, existierte zu keinem der beiden Betrachtungszeitpunkte eine eigene Schulungsabteilung, die standardisierte Seminare oder ähnliches anbot. Die Schulung wurde in der Regel jeweils von den die Implementierung durchführenden Mitarbeitern des Fallstudienunternehmens oder der Partnerunternehmen auf das jeweilige Unternehmen zugeschnitten und durchgeführt. Die Koordinationsformen stimmen daher für beide Betrachtungszeitpunkte mit denen bei der Wertschöpfungsstufe Implementierung beschriebenen überein.

Das Fallstudienunternehmen sah sich 2009 insbesondere bei beratungsintensiveren Projekten nicht nur als Lieferant des eigenen Softwareprodukts, sondern auch als Systemintegrator von Lösungen anderer Anbieter. Um die Kunden zufrieden zu stellen, wurde daher die Wartung nicht nur für die herstellereigenen Module, sondern auch für die zu Beginn des Kapitels erwähnten Module von verschiedenen Softwarepartnern, die in das System integriert werden konnten, sowie darüber hinaus für weitere, in der Rolle als Systemintegrator betreute Software intern und somit in der Koordinationsform Hierarchie durchgeführt. 2000 gab es die Wartung für die Module fremder Unternehmen in dieser Form noch nicht; die Wartung für die eigene Software wurde auch schon komplett intern und somit in der Koordinationsform Hierarchie durchgeführt.

Zusammenfassend ergeben sich für 2000 und 2009 die in Abbildung 3 dargestellten Ausprägungen der zwischenbetrieblichen Arbeitsteilung.

\subsection{Fallstudie 3}

Die Erhebung der Fallstudie fand in Form eines knapp zweistündigen telefonischen Interviews im April 2009 sowie eines anderthalbstündigen persönlichen Interviews im Mai 2009, jeweils mit einem Prokuristen des Fallstudienunternehmens, sowie auf Basis einer eingehenden Dokumentenanalyse statt. Das Produkt, dessen Wertschöpfungsstruktur betrachtet wurde, ist eine ERP-Software für Maschinenund Anlagenbauer sowie Automobilhersteller und -zulieferer, die sich aus einem ERP-Standard-Modul sowie verschiedenen Modulen für spezielle Probleme der 
genannten Zielbranchen zusammensetzt. Neben der aktuellen Situation wurde vom Unternehmen als Betrachtungszeitpunkt in der Vergangenheit der Zeitraum 1999-2001 festgelegt.

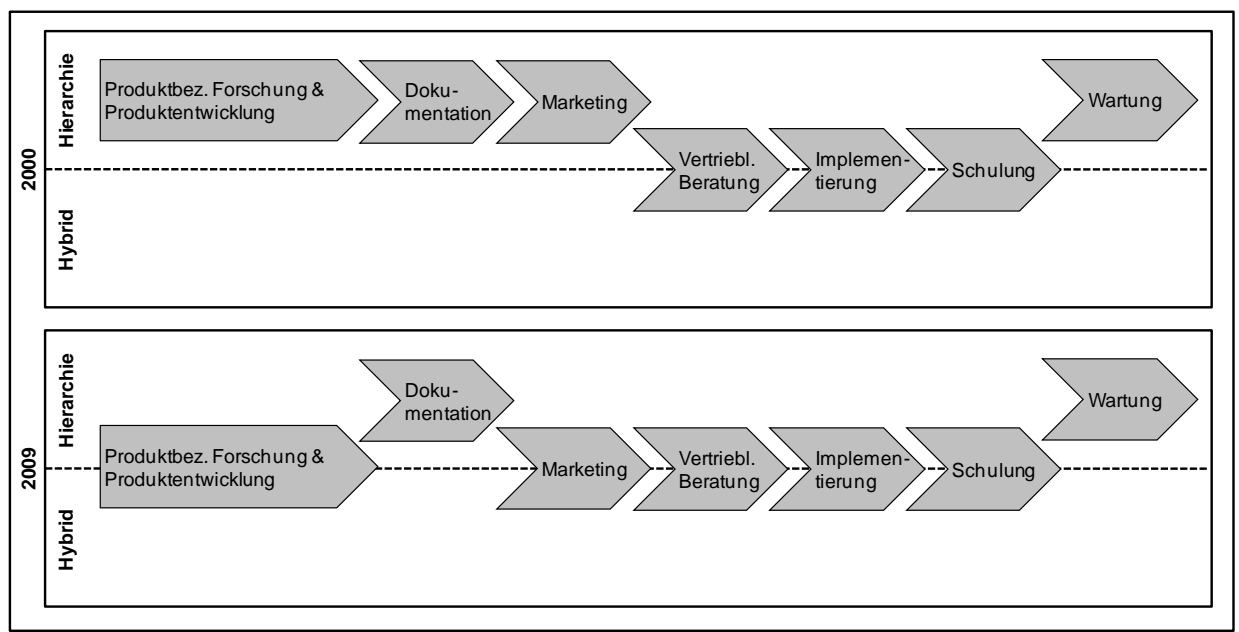

Abbildung 3: Zwischenbetriebliche Arbeitsteilung der Fallstudie 2 im Vergleich

Der Kern des Softwareprodukts ist vor rund 25 Jahren aus einem Forschungsprojekt hervorgegangen. Damals hatte das Fallstudienunternehmen in seiner Rolle als Softwarehaus gemeinsam mit Kunden ein PPS-System speziell für Auftragsfertiger gesucht. Ein entsprechendes System sei allerdings am Markt nicht vorhanden gewesen, sodass das Fallstudienunternehmen, die Kunden sowie Forschungseinrichtungen kooperiert hätten, um ein eigenes System zu spezifizieren, zu programmieren und zu implementieren. Diese Vorgehensweise führte das Fallstudienunternehmen bis auf den bereits vorhandenen Kern des Produkts auch 2009 bei produktbezogenem Forschungsbedarf durch, der sich rund um die Zielbranchen (Maschinen- und Anlagenbau; Automobilindustrie, insbesondere Zulieferer) ergab. Es wurden mit den Forschungseinrichtungen gemeinsam zusätzliche Lösungen aufgebaut und in den Produktstandard integriert. Auf Grund der strategischen Positionierung des Fallstudienunternehmens war die einzige Voraussetzung dabei, dass es sich um etwas handelt, was direkt mit dem Bereich Fertigungsprozesse zu tun hat. Wenn es um Make-or-Buy-Entscheidungen bei der Zusammenstellung des Produktportfolios ging, wurde grundsätzlich folgende Strategie verfolgt: Handelte es sich um ein Fertigungsthema, was die Position des Fallstudienunternehmens im Fertigungsumfeld stärken konnte, wurde die produktbezogene Forschung selbst durchgeführt. Dabei wurde entweder mit Kunden oder auch mit Forschungseinrichtungen kooperiert, wobei es sich jeweils um längerfristige partnerschaftlich angelegte Beziehungen mit einem ausgewählten Spektrum an Kunden bzw. Forschungseinrichtungen handelte, die sich auf Grund ihrer Branchenausrichtung oder 
lokalen Nähe anboten. 1999-2001 hingegen versuchte das Fallstudienunternehmen mit dem Softwareprodukt weniger den Spezialisierungsgedanken zu verfolgen, sondern einen deutlich breiteren Markt anzusprechen. Da die „Time to Market“ zu diesem Betrachtungszeitpunkt eine deutlich geringere war, führte das Fallstudienunternehmen die produktbezogene Forschung deutlich häufiger allein intern durch und musste sich stärker technischen Forschungsprojekten widmen. Hinsichtlich der idealtypischen Koordinationsformen lag auf der Wertschöpfungsstufe produktbezogene Forschung demnach sowohl 2009 als auch 1999-2001 sowohl hierarchische als auch hybride Koordination vor.

Die Produktentwicklung der Kernfunktionalitäten für den Bereich Fertigung wurde 2009 hauptsächlich intern durchgeführt, wobei der Mutterkonzern des Fallstudienunternehmens, um ressourcenschonend zu arbeiten, bestimmte übergeordnete Standardentwicklungen nach Polen ausgelagert hatte. Diese polnische Einheit war ein hundertprozentiges Tochterunternehmen des Mutterkonzerns und arbeitete ihren Schwestergesellschaften zu. Aus Sicht des Fallstudienunternehmens handelte es sich bei der von der polnischen Schwestergesellschaft durchgeführten Produktentwicklung jedoch um hybride Koordination. Des Weiteren wurden Funktionalitäten aus Randbereichen, in denen das Fallstudienunternehmen nicht tätig war (wie beispielsweise Dokumenten- oder Qualitätsmanagement) von ausgewählten Produktpartnern, die auf bestimmte Lösungsangebote spezialisiert waren, bezogen. Diese Ergänzungen des Funktionsumfangs wurden über Schnittstellen integriert, wobei das Fallstudienunternehmen bei Kundenprojekten als Generalunternehmer fungierte. Somit lag 2009 bezüglich der Kernproduktentwicklung sowohl hierarchische, als auch hybride (polnisches Schwesterunternehmen) Koordination und bezüglich der Entwicklung von Ergänzungen rein hybride (Produktpartner) Koordination vor. 1999-2001 erfolgte die Entwicklung des Kernprodukts hingegen ausschließlich intern, da es das diesbezügliche Outsourcing an das Schwesterunternehmen in Polen noch nicht gab. Allerdings wurden auch damals schon - sogar in einem größeren Maße als 2009 - ergänzenden Funktionalitäten von Drittanbietern bezogen: Es gab deutlich mehr Drittanbieter als 2009 und die Partnerschaft zu diesen war weniger eng, sodass häufig zur Realisation des Pflichtenhefts von Endkunden eine bestimmte Software nur einige wenige Male hinzugekauft und integriert wurde, was der idealtypischen Koordinationsform Markt entspricht. Deshalb ist für den Betrachtungszeitraum 1999-2001 wie folgt zu unterscheiden: Im Bereich der Kernproduktentwicklung lag ausschließlich hierarchische Koordination vor, im Bereich der Entwicklung ergänzender Funktionalitäten hingegen sowohl hybride als auch marktliche Koordination. Obwohl sich die Position der Teilaktivität „Ergänzungsentwicklung“ in unserer Darstellung daher von der Grenze zwischen den „Swimlanes“ Hybrid und Markt (1999-2001) auf die „Swimlane“ Hybrid (2009) verschiebt, bedeutet dies per definitionem keine Änderung des Grades an zwischenbetrieblicher Arbeitsteilung aus Sicht des Fallstudienunternehmens. Dieses war nämlich weder 1999-2001 noch 2009 an der Teilaktivität „Ergänzungsentwicklung“ beteiligt. 
Die Dokumentation wurde 2009 mittels Wikis realisiert und von eigenen Mitarbeitern aus dem Unternehmensbereich Marketing und somit in der Koordinationsform Hierarchie durchgeführt. Dabei konnten auch die Dokumentationen der in das Kernprodukt integrierten Drittprodukte in das Wiki aufgenommen werden. Auch 1999-2001 wurde die Dokumentation schon vollständig intern durchgeführt, allerdings noch nicht auf Wiki-Basis, sodass die Integration von Drittdokumentationen insbesondere in die Online-Hilfe oftmals zu Problemen führte.

Die Aktivitäten der Wertschöpfungsstufe Marketing wurden vom Fallstudienunternehmen zu beiden Betrachtungszeitpunkten nahezu vollständig intern und somit in der idealtypischen Koordinationsform Hierarchie durchgeführt.

Der Hauptzugang zum Kunden war zum Betrachtungszeitpunkt 2009 ein direkter Vertriebskanal, der mit der Komplexität sowohl der Anforderungen der Endkunden als auch des Produkts selbst begründet wurde. Da das Fallstudienunternehmen 1991-2001 einen breiteren Markt ansprechen wollte und dafür mehrere Vertriebskanäle benötigte, war neben dem direkten Vertrieb die indirekte Variante über Partnerunternehmen stark ausgeprägt. Zusammenfassend kann festgehalten werden, dass auf der Wertschöpfungsstufe vertriebliche Beratung 2009 die hierarchische Koordination dominierte. 1999-2001 hingegen lag neben hierarchischer in hohem Maße auch hybride Koordination vor.

2009 wurde die Wertschöpfungsstufe Implementierung im Kern vom Fallstudienunternehmen selbst durchgeführt, sodass die hierarchische Koordination dominierte. Ebenso wie bei der vertrieblichen Beratung spielten die Partnerunternehmen hingegen 1999-2001 auf Grund der gewollt breiteren Marktansprache eine größere Rolle, sodass auch hier sowohl hierarchische, als auch hybride Koordination vorlag.

Die Schulung wurde 2009 im Sinne des vom Fallstudienunternehmen verfolgten ganzheitlichen Ansatzes als zusätzliche Aktivität im Rahmen der Implementierung beim Kunden vor Ort durchgeführt. Die idealtypische Koordinationsform entspricht daher der bei der Implementierung dominierenden Hierarchie. 19992001 hingegen existierte auf Grund der deutlich breiteren Branchenausrichtung eine eigene Schulungsabteilung am Hauptsitz des Fallstudienunternehmens. Des Weiteren boten auch die in einer großen Anzahl vorhandenen Partnerunternehmen eigene Schulungen an, sodass sowohl hierarchische als auch hybride Koordination vorlag.

Das Fallstudienunternehmen sah die Aktivitäten der Wertschöpfungsstufe Wartung als wichtigste Erlösquelle von Standardsoftwareanbietern an. Diese wurden zu beiden Betrachtungszeitpunkten rein intern und somit in der Koordinationsform Hierarchie durchgeführt.

Zusammenfassend ergeben sich für 1999-2001 und 2009 die in Abbildung 4 dargestellten Ausprägungen der zwischenbetrieblichen Arbeitsteilung. 


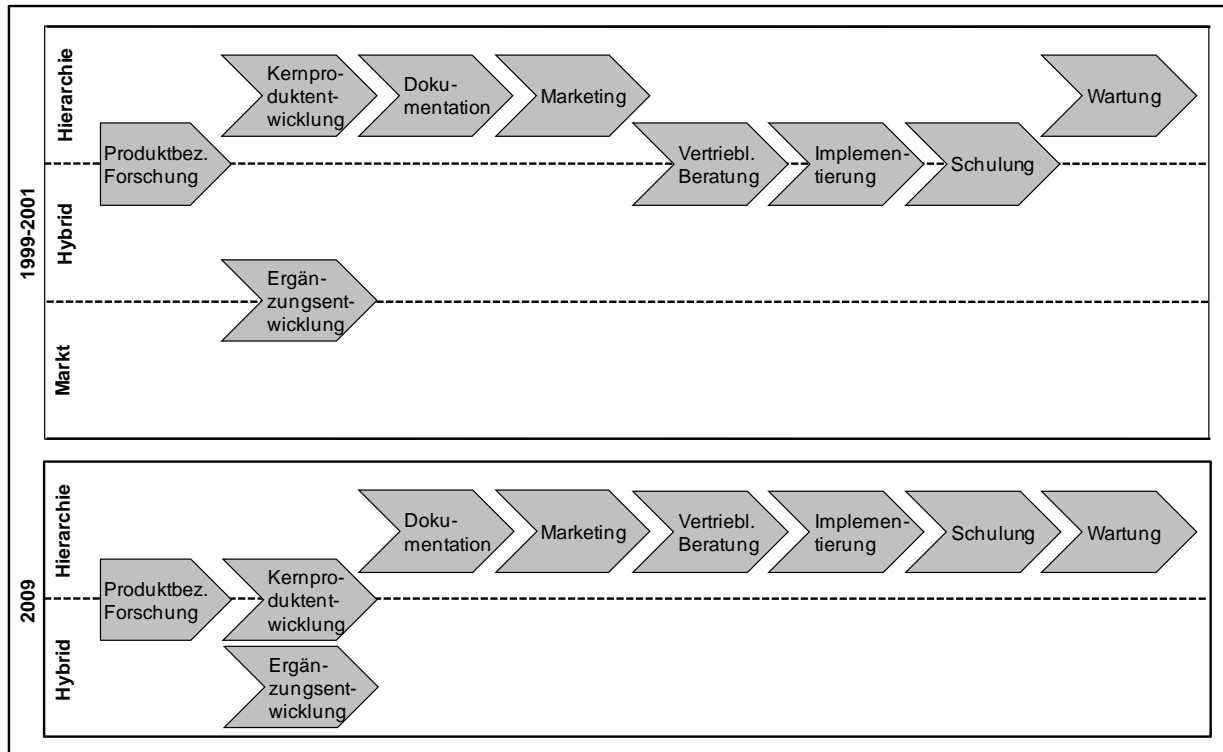

Abbildung 4: Zwischenbetriebliche Arbeitsteilung der Fallstudie 3 im Vergleich

\section{Zusammenfassung und Fazit}

In Abbildung 5 ist überblicksartig dargestellt, in welchen der von uns erhobenen Fallstudien es auf welchen Wertschöpfungsstufen aus Sicht des jeweiligen Fallstudienunternehmens zu einem vergleichsweise gestiegenen ( $)$ oder geringeren $(\Omega)$ Grad an zwischenbetrieblicher Arbeitsteilung gekommen ist, bzw. wo sich keine Veränderung ( $\boldsymbol{x})$ feststellen ließ.

Die Studie von Wolf et al. (2008) konnte eine zunehmende zwischenbetriebliche Arbeitsteilung vor allem auf den Downstream-Wertschöpfungsstufen von SAP feststellen. In der vorliegenden Untersuchung hingegen ergab sich tendenziell eine zunehmende zwischenbetriebliche Arbeitsteilung vor allem auf den UpstreamWertschöpfungsstufen produktbezogene Forschung (Fälle 1, 2) sowie Produktentwicklung (Fälle 1, 2, 3).

Ein im Zeitvergleich geringerer Grad an zwischenbetrieblicher Arbeitsteilung ergab sich - wiederum im Gegenteil zur erwähnten Studie - auf den DownstreamWertschöpfungsstufen vertriebliche Beratung (Fälle 1, 3), Implementierung (Fall 3) und Schulung (Fälle 1, 3). Bei Fallstudie 1 lässt sich die Veränderung von 2005 zu 2009 mit der hinzugekommenen Unterstützung der Vertriebspartner durch das Fallstudienunternehmen bei komplexeren und größeren Kundenpräsentationen im Rahmen der vertrieblichen Beratung sowie bei der fachlichen Schulung nach bestimmten neuen Releases erklären. Bei Fallstudie 3 hingegen ist die Reintegration der Wertschöpfungsstufen vertriebliche Beratung, Implementierung und Schulung auf eine strate- 
gisch motivierte Fokussierung des Zielmarktes und den damit verbundenen Wechsel zu einem direkten Vertriebsmodell zurückzuführen.

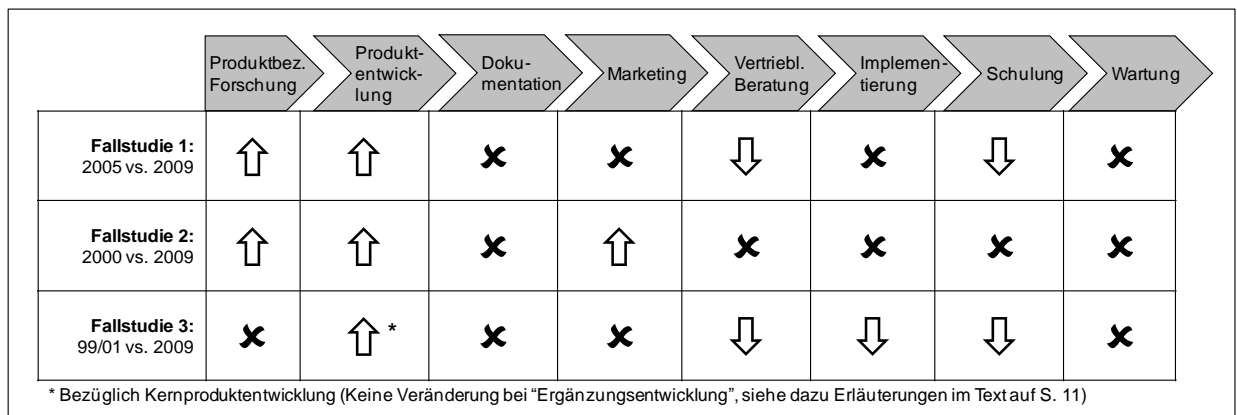

Abbildung 5: Fallstudienergebnisse im Überblick

Die festgestellten Unterschiede zur Vorgängerstudie lassen sich dahingehend interpretieren, dass wir mittelständische Softwarehersteller untersucht haben, die einen vergleichsmäßig kleineren Zielmarkt ansprechen. Deshalb mussten diese kein so enormes Wachstum bewältigen wie SAP und sie benötigten in geringerem Maße nicht zu den eigenen Kernkompetenzen zählendes spezifisches Wissen (wie z. B. länderspezifische Rechnungslegungsstandards), was die festgestellten Hauptgründe für die gestiegene zwischenbetriebliche Arbeitsteilung auf den DownstreamWertschöpfungsstufen von SAP war. Eine Industrialisierung durch zunehmende zwischenbetriebliche Arbeitsteilung ist somit zwar grundsätzlich auch in den von uns erhobenen Fallstudien festzustellen, allerdings ausschließlich im Rahmen des seit längerem bekannten Outsourcing der eigentlichen Softwareentwicklung im Sinne der Erstellung von Programmcode sowie der Kooperation mit Partnern oder Forschungseinrichtungen bei der produktbezogenen Forschung. Auf den anderen Wertschöpfungsstufen ergaben sich keine oder sogar gegenläufige Entwicklungen.

\section{Literatur}

Binner HF (1987) Anforderungsgerechte Datenermittlung für Fertigungssteuerungssysteme. Beuth, Köln.

Budäus D (1990) Industriebetriebe und Industriezweige: Kennzeichnung,

Systematisierung, Struktur und Entwicklungen. In Jacob H (Hrsg)

Industriebetriebslehre. Gabler, Wiesbaden.

Buxmann P, Diefenbach H, Hess T (2008) Die Softwareindustrie: Ökonomische

Prinzipien, Strategien, Perspektiven. Springer, Berlin.

Fischer J (2007) Industrialisierung der Software-Entwicklung. ZfCM

2007(Sonderheft 2): 74-82. 
Hess T, Buxmann P, Mann F, Königer M (2007) Industrialisierung der Softwarebranche: Erfahrungen deutscher Anbieter. Management Report Nr. 3/07 des Instituts für Wirtschaftsinformatik und Neue Medien. LMU, München.

Hildenbrand T, Rothlauf F, Heinzl A (2007) Ansätze zur kollaborativen Softwareentwicklung. WIRTSCHAFTSINFORMATIK 2007(Sonderheft): 7280.

Heinen E (1991) Industriebetriebslehre: Entscheidungen im Industriebetrieb. Gabler, Wiesbaden.

Kilian-Kehr R, Orestis T, Voelz D (2007) Industrialisation of the Software Sector. WIRTSCHAFTSINFORMATIK 2007(Sonderheft): 62-71.

Mertens P, Heinrich LJ (2002) Wirtschaftsinformatik: Ein interdisziplinäres Fach setzt sich durch. In Gaugler E, Köhler R (Hrsg) Entwicklung der Betriebswirtschaftslehre: 100 Jahre Fachdisziplin - zugleich eine Verlagsgeschichte. Schäffer-Pöschel, Stuttgart.

Mertens P, Bodendorf F, König W, Picot A, Schumann M, Hess T (2005) Grundzüge der Wirtschaftsinformatik. Springer, Heidelberg.

Meyer T, Stobbe A (2007) Offshoring - Welche "Standorte" wählen deutsche Unternehmen? WIRTSCHAFTSINFORMATIK 2007(Sonderheft): 81-89.

Picot A, Baumann O (2009) Die Bedeutung der Organisationstheorie für die Entwicklung der Wirtschaftsinformatik. WIRTSCHAFTSINFORMATIK 2009(1): 72-81.

Picot A, Reichwald R, Wigand RT (2008) Information, organization and management. Springer, Heidelberg.

Schweitzer M (1994) Industriebetriebslehre: Das Wirtschaften in Industriebetrieben. Vahlen, München.

Williamson OE (1991) Comparative economic organization: the analysis of discrete structural alternatives. Administrative Science Quarterly 36(2): 269296.

Yin RK (2003) Case study research - design and method. Sage, Thousand Oaks.

Wigand RT, Mertens P, Bodendorf F, König W, Picot A, Schumann M (2004) Introduction to business information systems. Springer, Heidelberg. 
Wolf CM, Geiger K, Benlian A, Hess T, Buxmann P (2008) Spezialisierung als Ausprägungsform einer Industrialisierung der Software-Branche - Eine Analyse am Beispiel der ERP-Software von SAP. In Herzwurm G, Mikusz M (Hrsg) Industrialisierung des Software-Managements. GI, Stuttgart.

Zäpfel G (1989) Taktisches Produktions-Management. De Gruyter, Berlin. 